\title{
EXIT Chart Aided Design of Near-Capacity Self-Concatenated Trellis Coded Modulation Using Iterative Decoding
}

\author{
Muhammad Fasih Uddin Butt, Soon Xin Ng and Lajos Hanzo \\ School of ECS, University of Southampton, SO17 1BJ, United Kingdom. \\ Tel: +44-23-8059 3125, Fax: +44-23-8059 4508 \\ Email: \{mfub06r, sxn, lh\}@ecs.soton.ac.uk, http://www-mobile.ecs.soton.ac.uk
}

\begin{abstract}
In this contribution we design the Iteratively Decoded Self-Concatenated Convolutional Codes (SECCC-ID) using Extrinsic Information Transfer (EXIT) charts. Good constituent Trellis Coded Modulation (TCM) codes are selected for communicating over both uncorrelated Rayleigh fading and Additive White Gaussian Noise (AWGN) channels. At the receiver iterative decoding is invoked for exchanging extrinsic information between the hypothetical decoder components. The convergence behaviour of the decoder is analysed with the aid of symbol-based EXIT charts. Similarly, the search for the best TCM constituent codes is also based on EXIT chart analysis. Finally, we demonstrate that the selected codes are capable of operating within $1 \mathrm{~dB}$ from the maximum achievable rate.
\end{abstract}

\section{INTRODUCTION}

TCM constitutes a joint coding and modulation technique proposed by Ungerböck [1], where a rate $n /(n+1)$ trellis code is combined with an $M=\left(2^{n+1}\right)$ - point signal constellation. It requires no bandwidth expansion relative to an uncoded $2^{n}$-point modulation scheme and yet, it is capable of achieving significant coding gains for transmission over power- and band-limited channels. Its applications to wireless communication channels has also been explored in [2] and [3], for example.

Concatenated coding schemes were first presented in [4]. Turbo codes based on parallel concatenated convolutional codes (PCCC) using two or more constituent codes were proposed in [5]. The discovery of turbo codes was a breakthrough in coding theory, because they are capable of operating near the Shannon limit [6]. Various turbo trellis coded modulation (TTCM) schemes were proposed in [7], [8] and [9]. Serially concatenated convolutional codes (SCCC) [10] have been shown to yield a performance comparable, and in some cases superior, to turbo codes. Self-concatenated convolutional codes (SECCC-ID) constitute another attractive iterative detection aided-code family proposed by Benedetto et al. [11] and Loeliger [12].

Extrinsic Information Transfer (EXIT) charts [13] constitute an excellent tool designed for analysing the convergence behaviour of an iterative decoding/detection scheme without performing time-consuming bit-by-bit decoding. Symbolbased EXIT charts [14] of non-binary serial and parallel concatenated schemes have been studied in [15] and [16],

\footnotetext{
The financial support of COMSATS Institute of Information Technology, Islamabad under the auspices of Higher Education Commission, Pakistan and that of the EPSRC UK is gratefully acknowledged.
}

respectively. However, EXIT charts have not been used for designing SECCC-ID schemes.

SECCCs exhibit a low complexity system since they invoke only a single encoder and a single decoder. The published studies in the literature of SECCC-ID schemes do not aim for finding good codes in terms of decoding convergence. An EXIT chart based analysis of the iterative decoder provides an insight into its decoding convergence behaviour and hence it is helpful for finding the best constituent TCM codes for SECCCs.

The rest of the paper is organised as follows. Section II presents our system model, while Section III discusses our code design procedure using EXIT charts. Our results are discussed in Section IV, while our conclusions are offered in Section V.

\section{SYSTEM MODEL}

We consider a half-rate SECCC scheme in this paper. Furthermore, $M=4$-ary Phase-Shift Keying (4PSK) modulation is used and both the Additive White Gaussian Noise (AWGN) and uncorrelated Rayleigh fading channels are considered.

As shown in Fig. 1, the input bit sequence $\left\{b_{1}\right\}$ of the self-concatenated encoder is interleaved for yielding the bit sequence $\left\{b_{2}\right\}$. The resultant bit sequences are input to the TCM constituent encoder. At the output of the encoder the interleaved bit sequence is punctured. Hence, the output of the encoder is composed of the combined systematic bit sequence and parity bit sequence.

The TCM constituent encoder has a coding rate of $R_{0}=\frac{2}{3}$, where two input bits, namely $b_{1}$ and $b_{2}$ are fed to the TCM encoder for generating three output bits, namely $b_{0}, b_{1}$ and $b_{2}$, during each encoding instance. However, the interleaved bit $b_{2}$ is punctured for attaining a higher rate of $R=\frac{1}{2}$ as compared to a $\frac{1}{3}$-rate, if bit $b_{2}$ was not punctured. The systematic and parity bits, $b_{0}$ and $b_{1}$, are mapped to a QPSK symbol as $x=$ $\mu\left(b_{0} b_{1}\right)$, where $\mu($.$) is the Set Partitioning (SP) based mapping$ function [2]. The QPSK symbol $x$ is then transmitted over the communication channel.

At the receiver side the received symbol is given by:

$$
y=h x+n,
$$

where $h$ is the channel's non-dispersive fading coefficient and $n$ is the AWGN having a variance of $\frac{N_{0}}{2}$ per dimension. This signal is then used by a soft demapper for calculating the 


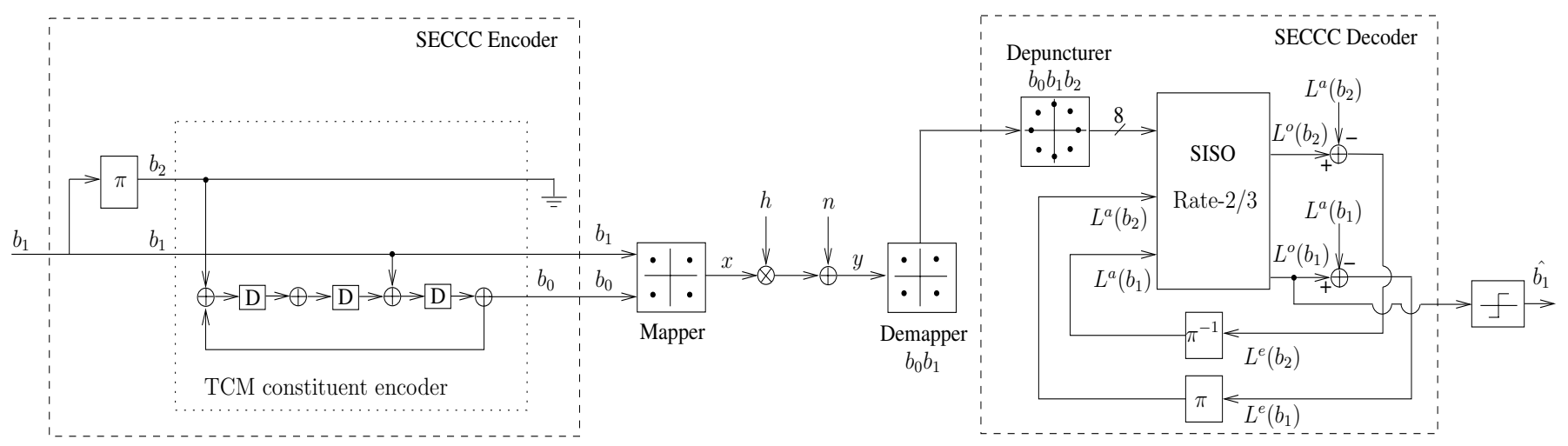

Fig. 1. SECCC-ID System

conditional probability density function (PDF) of receiving $y$, when $x^{(m)}$ was transmitted:

$$
P\left(y \mid x^{(m)}\right)=\frac{1}{\pi N_{0}} \exp \left(-\frac{\left|y-h x^{(m)}\right|^{2}}{N_{0}}\right),
$$

where $x^{(m)}=\mu\left(b_{0} b_{1}\right)$ is the hypothetically transmitted 4PSK symbol for $m \in\{0,1,2,3\}$. Then these PDFs are passed to a soft depuncturer for computing the conditional PDF of the $(n+1)=3$-bit coded symbol:

$$
P\left(y \mid \tilde{x}^{(l)}\right)=P\left(y \mid x^{(m)}\right) P\left(b_{2}\right),
$$

where $\tilde{x}^{(l)}$ is the hypothetically transmitted 3-bit symbol related to $b_{0}, b_{1}$ and $b_{2}$ for $l \in\{0,1 . ., 7\}$. Since $b_{2}$ was punctured, the probability of transmitting $b_{2}$ is given by

$$
P\left(b_{2}\right)=0.5,
$$

The $2^{3}=8$-valued PDF $P\left(y \mid \tilde{x}^{(l)}\right)$ characterising each 3-bit symbol is then passed to the SECCC decoder shown in Fig. 1.

The decoder is a self-concatenated decoder using a softinput soft-output (SISO) maximum a posteriori probability (MAP) algorithm [17]. It first calculates the extrinsic LogLikelihood Ratio (LLR) of the information bits, namely $L_{e}\left(b_{1}\right)$ and $L_{e}\left(b_{2}\right)$. Then they are appropriately interleaved to yield the a priori LLRs of the information bits, namely $L_{a}\left(b_{1}\right)$ and $L_{a}\left(b_{2}\right)$, as shown in Fig. 1. Self-concatenated decoding proceeds, until a fixed number of iterations is reached.

\section{Code Design}

\section{A. EXIT Charts}

EXIT charts constitute a powerful tool designed for analysing the convergence behaviour of concatenated codes without time-consuming bit-by-bit simulation of the actual system. They analyse the input/output mutual information characteristics of a SISO decoder by modelling the a priori LLRs and computing the corresponding mutual information of the extrinsic LLRs. However, the EXIT chart computation assumes the employment of a sufficiently long interleaver, where the LLRs may be rendered Gaussian distributed. The waterfall-like region in the BER curve of a concatenated code can be successfully predicted with the aid of EXIT charts.

The decoding model of the SECCC-ID scheme can be represented by Fig. 2. The information bit sequence is $\underline{U}$, which is encoded for yielding the coded symbol sequence $\underline{X}$. It is then transmitted over the communication channel and the received symbol sequence is given by $\underline{Y}$, which is then fed to the SISO SECCC decoder. The a priori channel models the $a$ priori probabilities of the information bit sequence $\underline{U}$ by $\underline{A}(U)$ and its interleaved version $\underline{W}$ by $\underline{A}(W)$. The SECCC SISO decoder then computes both the a posteriori bit probabilities $\underline{O}(U)$ and the extrinsic bit probabilities $\underline{E}(U)$ and $\underline{E}(W)$.

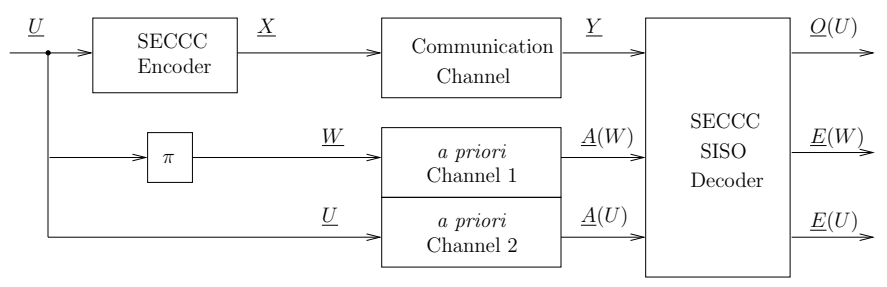

Fig. 2. Decoding model for an SECCC-ID scheme.

\section{B. Code Design Using EXIT Charts}

The EXIT chart based code design procedure can be explained using the example of a memory $\nu=3$ rate- $2 / 3 \mathrm{TCM}$ encoder as shown in Fig. 1. The connections shown at the encoder in Fig. 1 between the information bits and the modulo-2 adders are uniquely determined by the generator polynomials. The feed-forward generator polynomials are denoted as $g_{i}$ for $i \in\{1,2 \ldots, n\}$, while the feed-back generator polynomial is denoted as $g_{r}$. As shown in Fig. 1, there are 4 possible connection points, when there are three shift register stages, each denoted by $\mathrm{D}$. The four binary digits seen in the generator polynomials indicates the presence or absence of connections. For example, the generator polynomial corresponding to the first information bit $b_{1}$ is given by $g_{1}=[0010]_{2}$, which indicate that $b_{1}$ is connected only to the third modulo-2 adder from the left. The code generator is expressed in octal format as $G=\left[\begin{array}{lll}g_{r} & g_{1} & g_{2}\end{array}\right]_{8}=\left[\begin{array}{lll}11 & 2 & 4\end{array}\right]_{8}$.

Since we are not aiming for maximising the minimal distance, we can predefine the generator polynomial connections of the information bits and then only search for the best generator polynomial creating the parity bit [18]. The feedback generator polynomial is denoted as $g_{r}=[1 \times \mathrm{xx} 1]_{2}$, where the first and the last digits of $g_{r}$ are fixed to one and the 
rest of the two digits can be either $\mathrm{x}=0$ or $\mathrm{x}=1$, giving rise to only four possible devices for $g_{r}$ for the $\nu=3 \mathrm{TCM}$ code. These four feed-back generator polynomials are given by $\left[\begin{array}{lll}11 & 2 & 10\end{array}\right]_{8},\left[\begin{array}{lll}13 & 2 & 10\end{array}\right]_{8},\left[\begin{array}{lll}15 & 2 & 10\end{array}\right]_{8}$ and $\left[\begin{array}{lll}17 & 2 & 10\end{array}\right]_{8}$. We plot the corresponding EXIT curves for all these polynomials and then identify the specific code having the best decoding convergence by choosing the one that has an open EXIT tunnel at the lowest signal-to-noise ratio (SNR).

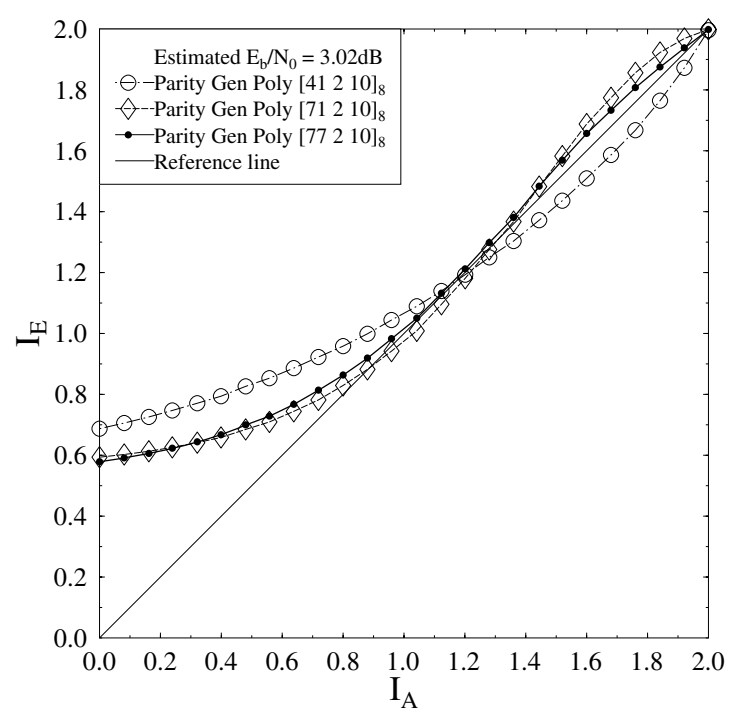

Fig. 3. Comparison of EXIT curves for various $\nu=5$ half-rate 4PSKassisted SECCC-ID codes at $E_{b} / N_{0}=3.02 \mathrm{~dB}$ using a block length of $10^{4}$ symbols.

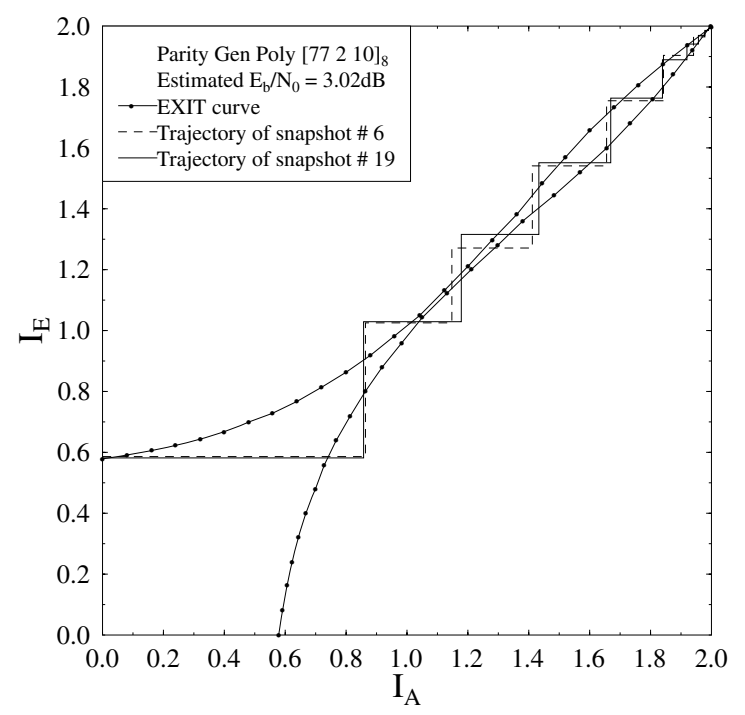

Fig. 4. EXIT chart and two snapshot decoding trajectories for half-rate 4PSK-assisted SECCC-ID using a block length of $10^{4}$ symbols and $\nu=5$ at $E_{b} / N_{0}=3.02 \mathrm{~dB}$.

The EXIT charts of self-concatenated codes are typically similar to those of the parallel concatenated TTCM schemes $[14,16]$, where an open EXIT tunnel exists if the EXIT curve does not intersect with the straight line connecting the point $\left(I_{A}=0, I_{E}=0\right)$ to the point $\left(I_{A}=2, I_{E}=2\right)$ in the EXIT chart. In [16] EXIT charts were successfully used to compare performance of different TTCM schemes by employing the same method to one of the component decoders.
The EXIT curves for three of the $\nu=5$ constituent TCM codes are shown in Fig. 3, where it was found that the code associated with the generator polynomial $\left[\begin{array}{lll}77 & 2 & 10\end{array}\right]_{8}$ has an open EXIT tunnel at the lowest $E_{b} / N_{0}$ value of $3.02 \mathrm{~dB}$. Fig. 4 depicts the EXIT curves of the constituent codes having a generator polynomial of $\left[\begin{array}{lll}77 & 2 & 10\end{array}\right]_{8}$ together with two of its decoding trajectory snapshots. The two EXIT curves are for two hypothetical decoder components of SECCC iterating between each other. Since these are identical components, therefore we need to compute the EXIT curve of only one component and the other is its mirror image. It is for the same reason that in Fig. 3 only one EXIT curve (of a particular generator polynomial) has been compared against a 45 degree diagonal line. The EXIT curves of the hypothetical decoder components are plotted on the same EXIT chart together with its decoding trajectory for the sake of visualizing the transfer of extrinsic information between the decoders. Similar to the EXIT curves of the TTCM schemes, the decoding trajectories based on bit-by-bit simulations do not exactly match the predicted EXIT curves [18]. The main reason for the mismatch is that the EXIT charts were generated based on the assumption that the extrinsic information and the systematic information of each TCM encoded symbol are independent of each other, which has a limited validity since both the systematic and the parity bits are transmitted together as a single $2^{n+1}$-ary symbol. For a symbol-based decoder, a mismatch between its decoding trajectory and its predicted EXIT curves is due to the correlation of the bits in each coded symbol $[14,16]$. However, we found that the EXIT charts of the SECCC scheme can be used as upper bounds since the actual EXIT chart tunnel is always wider than the predicted EXIT chart tunnel. Furthermore, the best TCM code found based on the EXIT charts also exhibits the best BER performance based on bit-by-bit simulations, as we will see in Section IV.

\section{RESUlts AND Discussion}

More quantitatively, the above mentioned EXIT chart method was used to find the best constituent TCM codes for $\nu=\{3,4,5\}$, when communicating over AWGN and uncorrelated Rayleigh fading channels. The corresponding generator polynomials and the channel capacity limit are shown in Table I together with the predicted and actual convergence thresholds expressed in $E_{b} / N_{0}$.

\begin{tabular}{|c|l|l|l|l|l|l|l|}
\hline$\nu$ & $\begin{array}{l}\text { Code } \\
\text { Polynomial } \\
\text { (Octal) }\end{array}$ & \multicolumn{2}{|l|}{$\begin{array}{l}\text { AWGN Channel } \\
E_{b} / N_{0}(\mathrm{~dB})\end{array}$} & \multicolumn{3}{|c|}{$\begin{array}{l}\text { Rayleigh Channel } \\
E_{b} / N_{0}(\mathrm{~dB})\end{array}$} \\
\cline { 3 - 8 } & & Pred. & Actual & $\omega$ & Pred. & Actual & $\omega$ \\
\hline \hline 3 & {$\left[\begin{array}{lll}17 & 2 & 10\end{array}\right]_{8}$} & 1.19 & 1.0 & 0.19 & 3.32 & 3.00 & 1.83 \\
4 & {$\left[\begin{array}{llll}37 & 2 & 10\end{array}\right]_{8}$} & 1.06 & 0.7 & & 3.09 & 2.70 & \\
5 & {$\left[\begin{array}{llll}77 & 2 & 10\end{array}\right]_{8}$} & 1.02 & 0.7 & & 3.02 & 2.60 & \\
\hline
\end{tabular}

TABLE I

THE CODE POLYNOMIALS OF THE BEST TCM CONSTITUENT CODES AND THEIR DECODING CONVERGENCE THRESHOLDS.

The predicted convergence threshold is based on the EXIT chart analysis as explained in Section III-B, while the actual 
convergence threshold is based on the corresponding BER curve given by the specified $E_{b} / N_{0}$ value, where there is a sudden drop of BER after a certain number of decoding iterations. It becomes possible to attain an infinitesimally low BER beyond the convergence threshold, provided that the block length is sufficiently long and the number of decoding iteration is sufficiently high. The BER versus $E_{b} / N_{0}$ performance curves of the various 4PSK-assisted SECCCID schemes recorded from symbol-by-symbol simulations are shown in Figs. 5, 6 and 7. A block length of $10^{4}$ symbols was considered and the number of decoding iterations $(I)$ was fixed to 20 in the simulations.

For the best constituent TCM codes at fixed code memory $\nu$, the distance from the channel capacity to their convergence threshold has been shown in Figs. 5, 6 and 7.

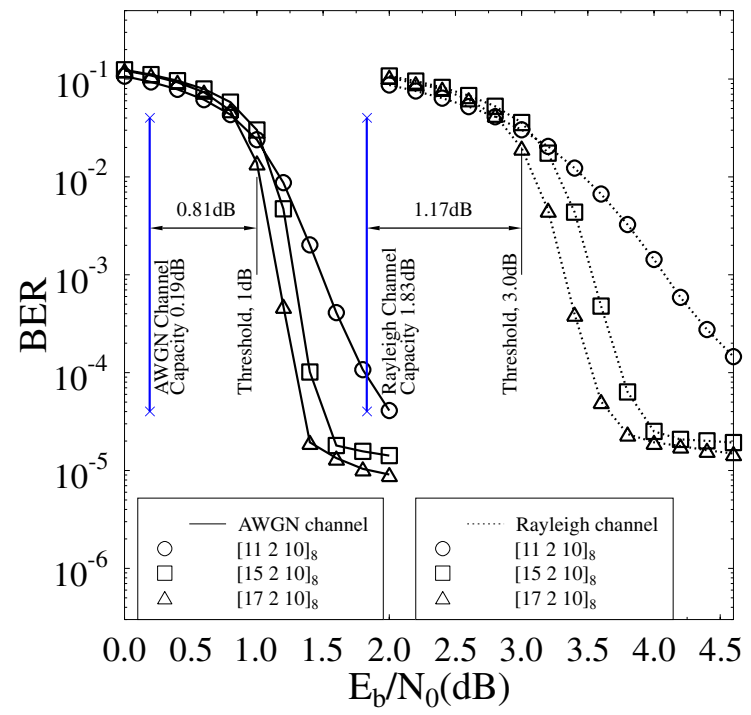

Fig. 5. The BER versus $E_{b} / N_{0}$ performance of various $\nu=3$ half-rate 4PSK-assisted SECCC-ID schemes when employing a block length of $10^{4}$ symbols and $I=20$ decoding iterations.

It can be observed that by increasing the code memory $\nu$ from 3 to 4 there is a $0.3 \mathrm{~dB}$ gain in case of both AWGN and uncorrelated Rayleigh fading channel. However, when $\nu$ is increased from 3 to 5 , the gain stays at $0.3 \mathrm{~dB}$ in case of the AWGN channel, but increases to $0.4 \mathrm{~dB}$ in case of the uncorrelated Rayleigh fading channel.

As we can see from Table I, the actual achievable convergence threshold is about $0.3 \mathrm{~dB}$ lower than the convergence threshold predicted by the EXIT chart. However, the best code found for a given code memory also exhibits the best BER performance among the top three codes considered for that particular code memory, as we can see from Figs. 5 to 7. Hence, the symbol-based EXIT chart is useful for finding the best TCM constituent codes, when designing SECCCID schemes for having a decoding convergence at the lowest possible $E_{b} / N_{0}$ value. However, we need a 'truncated' union bound analysis [19], if we aim for attaining a low BER floor. In general, a low BER floor can be attained by increasing the block length [19].

The $E_{b} / N_{0}$ values required for attaining a capacity of

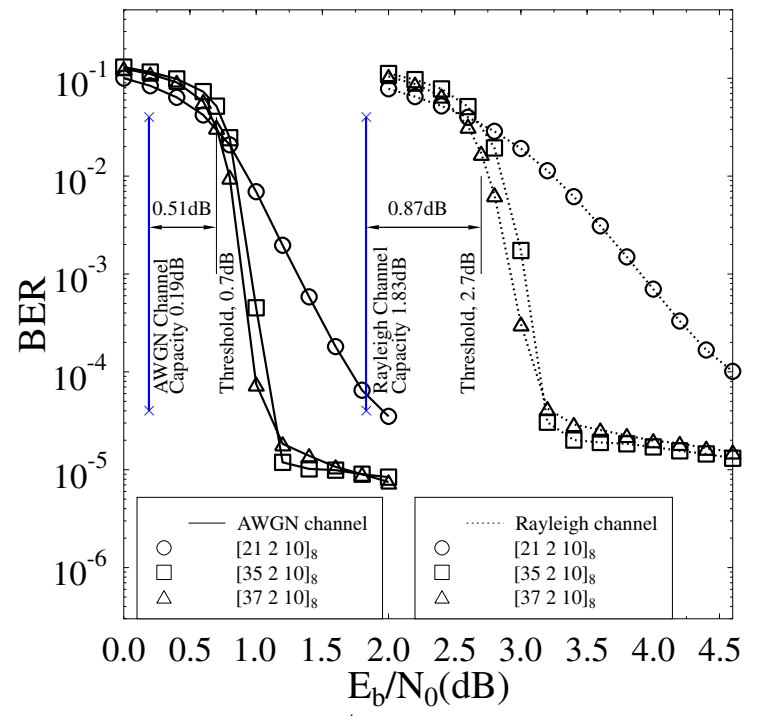

Fig. 6. The BER versus $E_{b} / N_{0}$ performance of various $\nu=4$ half-rate 4PSK-assisted SECCC-ID schemes when employing a block length of $10^{4}$ symbols and $I=20$ decoding iterations.

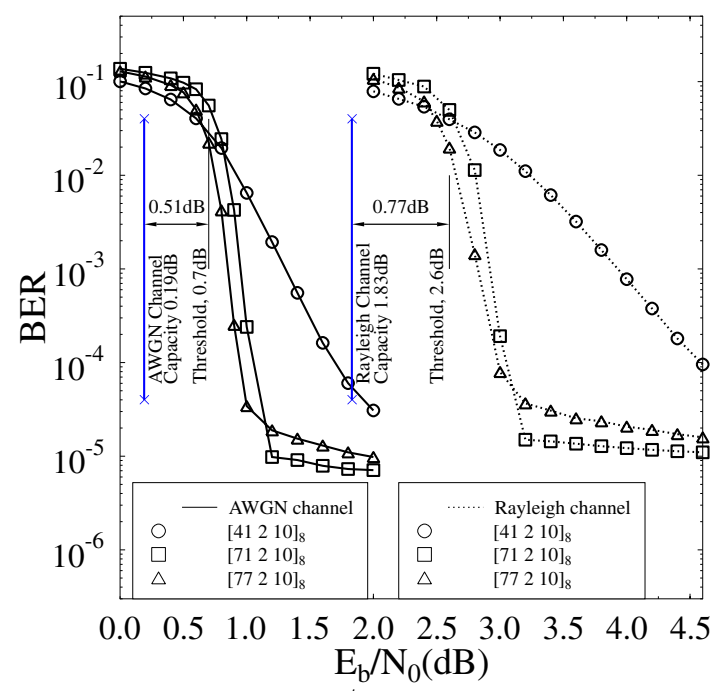

Fig. 7. The BER versus $E_{b} / N_{0}$ performance of various $\nu=5$ half-rate 4PSK-assisted SECCC-ID schemes when employing a block length of $10^{4}$ symbols and $I=20$ decoding iterations.

$1 \mathrm{bit} / \mathrm{s} / \mathrm{Hz}$ are $0.19 \mathrm{~dB}$ and $1.83 \mathrm{~dB}$ for the 4PSK-based discrete-input AWGN and Rayleigh fading channels, respectively [20]. As seen in Figs. 5 to 7, the codes found from the EXIT chart based design are capable of approaching the channel capacity of both the AWGN and uncorrelated Rayleigh fading channels. We then further increased the number of decoding iterations from $I=20$ to 50 and plotted the BER curves of best codes for $\nu=3,4$ and 5 in Fig. 8 .

When the number of iterations is increased from 20 to 50 , a further $0.1 \mathrm{~dB}$ gain is achieved by employing a generator polynomial of $\left[\begin{array}{lll}77 & 2 & 10\end{array}\right]_{8}$ for communicating over either the AWGN or uncorrelated Rayleigh fading channels, as seen in Figs. 7 and 8. Finally, we can see from Fig. 8, that the $\nu=5$ SECCC-ID scheme is only $0.41 \mathrm{~dB}$ and $0.67 \mathrm{~dB}$ away from the AWGN and Rayleigh fading channel's capacity, respectively. 


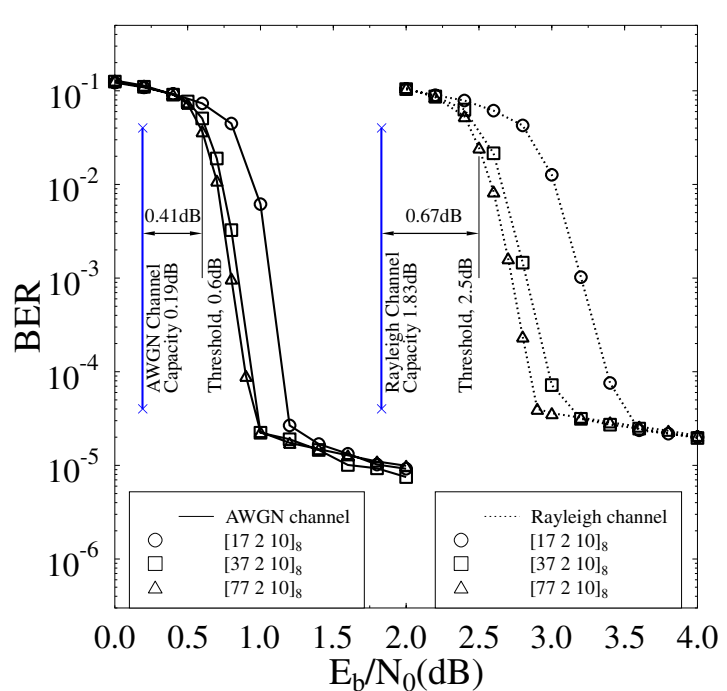

Fig. 8. The BER versus $E_{b} / N_{0}$ performance of best codes among $\nu=3,4$ and 5, half-rate 4PSK-assisted SECCC-ID schemes when employing a block length of $10^{4}$ symbols and $I=50$ decoding iterations.

\section{Conclusions}

We have designed near-capacity SECCC-ID schemes based on their decoding convergence analysis. The symbol-based EXIT chart was found to be fairly accurate in predicting the decoding convergence threshold, despite the inaccurate simplifying assumption that the extrinsic information and the systematic information of each SECCC-encoded symbol are independent of each other, which again has a limited validity. Good constituent TCM codes were found for assisting the SECCC-ID scheme in attaining decoding convergence at the lowest possible $E_{b} / N_{0}$ value, when communicating over both AWGN and uncorrelated Rayleigh fading channels. The SECCC-ID schemes designed are capable of operating within about $0.5 \mathrm{~dB}$ and $1.0 \mathrm{~dB}$ from the AWGN and Rayleigh fading channel's capacity, respectively. Our future work will focus on investigating the performance of SECCC-ID scheme in non-coherently detected cooperative communication systems as well as on designing other near-capacity self-concatenated codes using different constituent schemes.

\section{REFERENCES}

[1] G. Ungerböck, "Channel coding with multilevel/phase signals," IEEE Transactions on Information Theory, vol. 28, pp. 55-67, Jan. 1982.

[2] D. Divsalar and M. K. Simon, "The design of trellis coded MPSK for fading channels: Set partitioning for optimum code design," IEEE Transactions on Communications, vol. 36, pp. 1013-1021, Sept. 1988.

[3] D. Divsalar and M. K. Simon, "The design of trellis coded MPSK for fading channels: Performance criteria," IEEE Transactions on Communications, vol. 36, pp. 1004-1012, Sept. 1988.

[4] G. Forney, Concatenated Codes. MIT Press, Cambridge, MA,, 1966.

[5] C. Berrou, A. Glavieux, and P. Thitimajshima, "Near Shannon limit error correcting coding and decoding: Turbo codes," IEEE Transactions on Communications, pp. 1064-1070, 1993.

[6] C. Shannon, "A mathematical theory of communication," Bell Syst. Tech. Journal, vol. 27, pp. 623-656, 1948.

[7] S. Le Goff, A. Glavieux, and C. Berrou, "Turbo-codes and high spectral efficiency modulation," in IEEE International Conference on Communications, (New Orleans, LA, USA), pp. 645-649, May 1994.
[8] S. Benedetto, D. Divsalar, G. Montorsi, and F. Pollara, "Bandwidth efficient parallel concatenated coding schemes," Electronics Letters, vol. 31, pp. 2067-2069, Nov. 1995.

[9] P. Robertson and T. Worz, "Coded modulation scheme employing turbo codes," Electronics Letters, vol. 31, pp. 1546-1547, Aug. 1995.

[10] S. Benedetto, D. Divsalar, G. Montorsi, and F. Pollara, "Serial concatenated trellis coded modulation with iterative decoding," in IEEE International Symposium on Information Theory, (Ulm), p. 8, June/July 1997.

[11] S. Benedetto, D. Divsalar, G. Montorsi, and F. Pollara, "Selfconcatenated trellis coded modulation with self-iterative decoding," in IEEE Global Telecommunications Conference, vol. 1, (Sydney, NSW, Australia), pp. 585-591, 1998.

[12] H. A. Loeliger, "New turbo-like codes," in IEEE International Symposium on Information Theory, (Ulm), p. 109, June/July 1997.

[13] S. ten Brink, "Convergence behavior of iteratively decoded parallel concatenated codes," IEEE Transactions on Communications, vol. 49, pp. 1727-1737, Oct. 2001.

[14] J. Kliewer, S. X. Ng, and L. Hanzo, "Efficient computation of EXIT functions for nonbinary iterative decoding," IEEE Transactions on Communications, vol. 54, pp. 2133-2136, Dec. 2006.

[15] A. Grant, "Convergence of non-binary iterative decoding," in IEEE Global Telecommunications Conference, vol. 2, (San Antonio, TX, USA), pp. 1058-1062, Nov. 2001.

[16] H. Chen and A. Haimovich, "EXIT charts for turbo trellis-coded modulation," IEEE Communications Letters, vol. 8, pp. 668-670, Nov. 2004.

[17] S. Benedetto, D. Divsalar, G. Motorsi, and F. Pollara, "A soft-input softoutput APP module for iterative decoding of concatenated codes," IEEE Communications Letter, vol. 1, pp. 22-24, January 1997.

[18] S. X. Ng, J. Kliewer, O. R. Alamri, and L. Hanzo, "On the design of turbo trellis coded modulation schemes using symbol-based exit charts," in Vehicular Technology Conference, 2006. VTC-2006 Fall. 2006 IEEE 64th, pp. 1-5, Sept. 2006

[19] S. X. Ng, O. Alamri, Y. Li, and L. Hanzo, "Near-capacity turbo trellis coded modulation design," in Vehicular Technology Conference, 2007. VTC-2007 Fall. 2007 IEEE 66th, (Baltimore, MD, USA), pp. 17071711, Sept./Oct. 2007.

[20] L. Hanzo, S. X. Ng, T. Keller, and W. Webb, Quadrature amplitude modulation: From basics to adaptive trellis-coded, turbo-equalised and space-time coded OFDM, CDMA and MC-CDMA systems, pp. 746-748. Wiley-IEEE Press, 2nd ed., December 15, 2004. 